Pacific

Journal of

Mathematics

\title{
CROSSED PRODUCT ALGEBRAS AND DIRECT INTEGRAL DECOMPOSITION FOR LIE SUPERGROUPS
}

KARL-HERMANN NEEB AND HADi SALMASIAN

Volume 282 No. 1

May 2016 


\title{
CROSSED PRODUCT ALGEBRAS AND DIRECT INTEGRAL DECOMPOSITION FOR LIE SUPERGROUPS
}

\author{
KARL-HERMANN NEEB AND HADI SALMASIAN
}

\begin{abstract}
For every finite dimensional Lie supergroup $(G, \mathfrak{g})$, we define a $C^{*}$-algebra $\mathcal{A}:=\mathcal{A}(G, \mathfrak{g})$ and show that there exists a canonical bijective correspondence between unitary representations of $(G, \mathfrak{g})$ and nondegenerate $*$-representations of $\mathcal{A}$. The proof of existence of such a correspondence relies on a subtle characterization of smoothing operators of unitary representations previously studied by Neeb, Salmasian, and Zellner.

For a broad class of Lie supergroups, which includes nilpotent as well as classical simple ones, we prove that the associated $C^{*}$-algebra is CCR. In particular, we obtain the uniqueness of direct integral decomposition for unitary representations of these Lie supergroups.
\end{abstract}

\section{Introduction}

Unitary representations of Lie supergroups play an important role in the mathematical theory of supersymmetric quantum mechanics. One distinguished example of the role of these unitary representations is the classification of free relativistic superparticles (see [Ferrara et al. 1981] and [Salam and Strathdee 1974]), where a super analogue of the little group method of Mackey and Wigner is used.

Although the super version of the Mackey-Wigner method was used in the physics literature as early as the 1970 s, the problem of mathematical validity of this method in the context of supergroups was not addressed until less than a decade ago. This was done in [Carmeli et al. 2006], where the authors remedy this issue by laying the mathematically rigorous foundations of the analytic theory of unitary representations of Lie supergroups, using the equivalence of categories between the category of Lie supergroups and the category of Harish-Chandra pairs [Deligne and Morgan 1999, Section 3.8; Kostant 1977, Section 3.2]. The Harish-Chandra pair description of Lie supergroups will be explained in Definition 2.1 below.

MSC2010: 17B15, 22E45, 47L65.

Keywords: crossed product algebras, unitary representations, Lie supergroups, Harish-Chandra pairs, direct integral decomposition, CCR algebras. 
The groundwork laid in [Carmeli et al. 2006] has spawned research on the harmonic analysis of Lie supergroups. In particular, in [Salmasian 2010] the irreducible unitary representations of a nilpotent Lie supergroup are classified using an extension of Kirillov's orbit method (see also [Neeb and Salmasian 2011, Section 8]). For Lie supergroups corresponding to basic classical Lie superalgebras [Musson 2012, Definition 1.14], the irreducible unitary representations are indeed highest weight modules [Neeb and Salmasian 2011, Section 7], and therefore they are completely classified by the work done in [Jakobsen 1994].

The goal of this paper is to systematically study disintegration of arbitrary unitary representations of Lie supergroups into direct integrals of irreducible representations. To this end, for every finite dimensional Lie supergroup $(G, \mathfrak{g})$ we construct a $C^{*}$-algebra $\mathcal{A}:=\mathcal{A}(G, \mathfrak{g})$ whose nondegenerate $*$-representations are in bijective correspondence with unitary representations of $(G, \mathfrak{g})$. The $C^{*}$-algebra $\mathcal{A}$ is obtained as the completion of a crossed product $*$-algebra $\mathcal{A}^{\circ}$ that is associated to the action of $G$ by left translation on the convolution algebra of test functions. Here, indeed, it will be more convenient to replace $G$ by a slightly larger group $G_{\varepsilon} \cong G \times\{1, \varepsilon\}$, as the action of the extra element $\boldsymbol{\varepsilon}$ will automatically keep track of the $\mathbb{Z}_{2}$-grading of the representation space. Starting from a unitary representation $\left(\pi, \rho^{\pi}, \mathscr{H}\right)$ of $(G, \mathfrak{g})$, we obtain a representation of $\mathcal{A}$ by first extending $\left(\pi, \rho^{\pi}, \mathscr{H}\right)$ canonically to $\mathcal{A}^{\circ}$, and then uniquely to a nondegenerate $*$-representation $(\hat{\pi}, \mathscr{H})$ of $\mathcal{A}$ by continuity. Nevertheless, the construction of a representation of $(G, \mathfrak{g})$ from a representation $(\hat{\pi}, \mathscr{H})$ of $\mathcal{A}$ is more subtle, because the standard method of extending $(\hat{\pi}, \mathscr{H})$ to the multiplier algebra $M(\mathcal{A})$ does not work. Indeed, the Lie superalgebra $\mathfrak{g}$ does not act on $\mathcal{A}$ by multipliers. To circumvent this issue, we use the extension of $(\hat{\pi}, \mathscr{H})$ to the multiplier algebra $M\left(\mathcal{A}^{\circ}\right)$, and use the fact that $G$ and $\mathfrak{g}$ act on $\mathcal{A}^{\circ}$ through $M\left(\mathcal{A}^{\circ}\right)$. To complete the construction of the unitary representation of $(G, \mathfrak{g})$, we need to show that the action of $\mathfrak{g}$ is indeed defined on $\mathscr{H}^{\infty}$. To this end, we prove that $\hat{\pi}\left(\mathcal{A}^{\circ}\right) \mathscr{H}=\mathscr{H}^{\infty}$, where $\mathscr{H}^{\infty}$ denotes the space of smooth vectors of the action of $G$ on $\mathscr{H}$. The proof of the latter statement requires the Dixmier-Malliavin theorem [1978] and a subtle result from [Neeb et al. 2015, Theorem 2.11] on the characterization of smoothing operators of unitary representations, that is, operators $A: \mathscr{H} \rightarrow \mathscr{H}$ which map $\mathscr{H}$ into $\mathscr{H}^{\infty}$.

By the standard machinery of $C^{*}$-algebras [Dixmier 1974], statements on the existence and uniqueness of disintegration of nondegenerate $*$-representations of $\mathcal{A}$ can be transformed to similar statements on direct integral decompositions of unitary representations of $(G, \mathfrak{g})$. To obtain uniqueness of disintegration, it suffices to know that $\mathcal{A}$ is CCR, that is, the image of every irreducible $*$-representation of $\mathcal{A}$ lies in the algebra of compact operators. (Such $C^{*}$-algebras are sometimes called liminal.) We prove that $\mathcal{A}$ is CCR for a broad class of Lie supergroups, which includes nilpotent Lie supergroups as well as those which correspond to classical simple Lie 
superalgebras (see [Musson 2012, Section 1.3]). Therefore, for the aforementioned classes of Lie supergroups, one obtains uniqueness of disintegration of unitary representations.

This article is organized as follows. Section 2 is devoted to definitions and basic properties of unitary representations that will be used in the rest of the paper. In Section 3 we define the crossed product $*$-algebra $\mathcal{A}^{\circ}$. In Section 4 we construct the $C^{*}$-algebra $\mathcal{A}:=\mathcal{A}(G, \mathfrak{g})$ as the completion of the crossed product algebra $\mathcal{A}^{\circ}$. In Section 5 we prove that under the $G$-action on $\mathcal{A}$, orbit maps of elements of $\mathcal{A}^{\circ}$ are smooth. In Section 6 we describe the canonical bijective correspondence between unitary representations of the Lie supergroup $(G, \mathfrak{g})$ and the (ungraded) nondegenerate $*$-representations of $\mathcal{A}$. Finally, in Section 7 we give our liminality results for $C^{*}$-algebras of a broad class of Lie supergroups, including the nilpotent and classical simple ones.

\section{Basic definitions}

We begin with a rapid review of Lie supergroups (from the Harish-Chandra pair viewpoint) and their unitary representations. For a more elaborate reference, see [Carmeli et al. 2006].

Throughout this paper, $\mathbb{Z} / 2 \mathbb{Z}:=\{\overline{0}, \overline{1}\}$ denotes the field with two elements. If $V=V_{\overline{0}} \oplus V_{\overline{1}}$ is a $\mathbb{Z} / 2 \mathbb{Z}$-graded vector space, then the parity of a homogeneous element $x \in V$ is denoted by $|x| \in \mathbb{Z} / 2 \mathbb{Z}$.

Definition 2.1. A Lie supergroup is an ordered pair $(G, \mathfrak{g})$ with the following properties.

(i) $G$ is a Lie group.

(ii) $\mathfrak{g}=\mathfrak{g}_{\overline{0}} \oplus \mathfrak{g}_{\overline{1}}$ is a Lie superalgebra over $\mathbb{R}$.

(iii) $\mathfrak{g}_{0}^{-}$is the Lie algebra of $G$.

(iv) There exists a group homomorphism $\operatorname{Ad}: G \rightarrow \operatorname{Aut}(\mathfrak{g})$, defining a smooth action $G \times \mathfrak{g} \rightarrow \mathfrak{g}$, such that

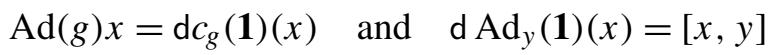

for every $x \in \mathfrak{g}_{\overline{0}}, y \in \mathfrak{g}$, and $g \in G$, where the map $c_{g}: G \rightarrow G$ is defined by $c_{g}\left(g^{\prime}\right):=g g^{\prime} g^{-1}$ and $\operatorname{Ad}_{y}: G \rightarrow \mathfrak{g}$ is defined by $\operatorname{Ad}_{y}(g):=\operatorname{Ad}(g) y$.

We assume that $\operatorname{dim} \mathfrak{g}<\infty$ and that the component group $G / G^{\circ}$ is finite. The Lie supergroup $(G, \mathfrak{g})$ is called connected if $G$ is a connected Lie group.

Remark 2.2. Here we should clarify that the condition given in Definition 2.1(iv) is identical to the ones given in our previous papers [Neeb and Salmasian 2013b, Definition 4.6.3(iv)] and [Neeb and Salmasian 2013a, Definition 7.1(iv)]. More 
precisely, in those two papers we tacitly assume that Ad is an extension of the adjoint action of $G$ on $\mathfrak{g}_{\overline{0}}$.

Let $(\pi, \mathscr{H})$ be a unitary representation of a Lie group $G$. For $x \in \operatorname{Lie}(G)$ and $v \in \mathscr{H}$, we set

$$
\mathrm{d} \pi(x) v:=\lim _{t \rightarrow 0} \frac{1}{t}\left(\pi\left(e^{t x}\right) v-v\right),
$$

whenever the limit exists. Here $e^{t x}:=\exp (t x)$ denotes the exponential map of $G$.

Definition 2.3. Let $(G, \mathfrak{g})$ be a Lie supergroup. A unitary representation of $(G, \mathfrak{g})$ is a triple $\left(\pi, \rho^{\pi}, \mathscr{H}\right)$ which satisfies the following properties.

(i) $\mathscr{H}$ has a $\mathbb{Z} / 2 \mathbb{Z}$-grading, that is, $\mathscr{H}=\mathscr{H}_{0} \oplus \mathscr{H}$, and $(\pi, \mathscr{H})$ is a smooth unitary representation of $G$ such that $\pi(g)$ preserves the $\mathbb{Z} / 2 \mathbb{Z}$-grading of $\mathscr{H}$ for every $g \in G$.

(ii) $\rho^{\pi}: \mathfrak{g} \rightarrow \operatorname{End}_{\mathbb{C}}\left(\mathscr{H}^{\infty}\right)$ is a representation of the Lie superalgebra $\mathfrak{g}$, where $\mathscr{H}^{\infty}=\mathscr{H}_{\overline{0}}^{\infty} \oplus \mathscr{H}_{\overline{1}}^{\infty}$ is the subspace consisting of all $v \in \mathscr{H}_{\text {for }}$ which the orbit map $G \rightarrow \mathscr{H}, g \mapsto \pi(g) v$ is smooth.

(iii) $\rho^{\pi}(x)=\left.\mathrm{d} \pi(x)\right|_{\mathscr{H}}$ for every $x \in \mathfrak{g}_{\overline{0}}$.

(iv) For every $x \in \mathfrak{g}_{\overline{1}}$, the operator $e^{-\pi i / 4} \rho^{\pi}(x)$ is symmetric. That is,

$$
-i \rho^{\pi}(x) \subseteq \rho^{\pi}(x)^{*} .
$$

(v) $\pi(g) \rho^{\pi}(x) \pi(g)^{-1}=\rho^{\pi}(\operatorname{Ad}(g) x)$ for every $g \in G$ and every $x \in \mathfrak{g}_{1}$.

Remark 2.4. By [Neeb and Salmasian 2013a, Proposition 6.13], the condition given in Definition 2.3(v) follows from the weaker condition that, for every element of the component group $G / G^{\circ}$, there exists a coset representative $g \in G$ such that

$$
\pi(g) \rho^{\pi}(x) \pi\left(g^{-1}\right)=\rho^{\pi}(\operatorname{Ad}(g) x) \text { for every } x \in \mathfrak{g} .
$$

Remark 2.5. As in [Neeb and Salmasian 2013b, Definition 6.7.1], a unitary representation $\left(\pi, \rho^{\pi}, \mathscr{H}\right)$ is called cyclic if there exists a vector $v \in \mathscr{H}_{\overline{0}}^{\infty}$ such that $\pi(G) \rho^{\pi}\left(\boldsymbol{U}\left(\mathfrak{g}_{\mathbb{C}}\right)\right) v$ spans a dense subspace of $\mathscr{H}$, where $\mathfrak{g}_{\mathbb{C}}:=\mathfrak{g} \otimes_{\mathbb{R}} \mathbb{C}$ and where $\boldsymbol{U}\left(\mathfrak{g}_{\mathbb{C}}\right)$ denotes the universal enveloping algebra of $\mathfrak{g}_{\mathbb{C}}$. A standard Zorn lemma argument shows that every unitary representation can be written as a direct sum of representations which are cyclic up to parity change. Furthermore, in [Neeb and Salmasian 2013b, Theorem 6.7.5] a Gelfand-Naimark-Segal construction is given which results in a correspondence between cyclic unitary representations and positive definite superfunctions of $(G, \mathfrak{g})$.

Let $\left(\pi, \rho^{\pi}, \mathscr{H}\right)$ be a unitary representation of $(G, \mathfrak{g})$. We equip the space $\mathscr{H}^{\infty}$ with the Fréchet topology induced by the seminorms $v \mapsto\|\mathrm{d} \pi(D) v\|$, for all $D \in \boldsymbol{U}\left(\mathfrak{g}_{\overline{0}}\right)$. This topology makes $\mathscr{H}^{\infty}$ a Fréchet space. 
Proposition 2.6. For every $x \in \mathfrak{g}$, the map $\rho^{\pi}(x): \mathscr{H}^{\infty} \rightarrow \mathscr{H}^{\infty}$ is continuous.

Proof. Continuity for $x \in \mathfrak{g}_{0}$ is standard, and therefore we will assume that $x \in \mathfrak{g}_{\overline{1}}$. We need to prove that, for every $x_{1}, \ldots, x_{\ell} \in \mathfrak{g}_{0}$, for $D:=x_{1} \cdots x_{\ell} \in \boldsymbol{U}\left(\mathfrak{g}_{0}\right)$, the map

$$
\mathscr{H}^{\infty} \rightarrow \mathbb{R}, \quad v \mapsto\left\|\mathrm{d} \pi(D) \rho^{\pi}(x) v\right\|
$$

is continuous at $0 \in \mathscr{H}^{\infty}$. First assume that $\ell=0$, so that $D=1 \in \boldsymbol{U}\left(\mathfrak{g}_{\overline{0}}\right)$. In this case, continuity of (1) follows from the inequality

$$
\left\|\rho^{\pi}(x) v\right\|^{2}=\left|\left\langle v, \rho^{\pi}(x)^{2} v\right\rangle\right| \leq \frac{1}{2}\|v\| \cdot\|\mathrm{d} \pi([x, x]) v\|
$$

and the definition of the topology of $\mathscr{H}^{\infty}$. To prove continuity of (1) for $\ell \geq 1$, we use the relation

$$
x_{1} \cdots x_{\ell} x=x x_{1} \cdots x_{\ell}+\sum_{i=1}^{\ell} x_{1} \cdots x_{i-1}\left[x_{i}, x\right] x_{i+1} \cdots x_{\ell}
$$

and induction on $\ell$.

Definition 2.7. A multiplier of an associative algebra $\mathcal{A}$ is a pair $(\lambda, \rho)$ of linear maps $\mathcal{A} \rightarrow \mathcal{A}$ which satisfy the relations

$$
\lambda(a b)=\lambda(a) b, \quad \rho(a b)=a \rho(b), \quad \text { and } \quad a \lambda(b)=\rho(a) b
$$

for every $a, b \in \mathcal{A}$.

If $\mathcal{A}$ is a $*$-algebra, then the multipliers of $\mathcal{A}$ form a $*$-algebra, denoted by $M(\mathcal{A})$, with multiplication and involution defined by

$$
(\lambda, \rho)\left(\lambda^{\prime}, \rho^{\prime}\right):=\left(\lambda \lambda^{\prime}, \rho^{\prime} \rho\right) \text { and }(\lambda, \rho)^{*}:=\left(\rho^{*}, \lambda^{*}\right),
$$

where $\lambda^{*}(a):=\lambda\left(a^{*}\right)^{*}$ and $\rho^{*}(a)=\rho\left(a^{*}\right)^{*}$.

\section{The crossed product $*$-algebra $\mathcal{A}^{\circ}$}

Fix a Lie supergroup $(G, \mathfrak{g})$. Set $G_{\boldsymbol{\varepsilon}}:=G \times\{\mathbf{1}, \boldsymbol{\varepsilon}\}$ such that $\boldsymbol{\varepsilon}^{2}=\mathbf{1}$, and define $\operatorname{Ad}(\varepsilon) x:=(-1)^{|x|} x$ for every homogeneous $x \in \mathfrak{g}$. We endow $G_{\varepsilon}$ with the product topology. Clearly $\left(G_{\varepsilon}, \mathfrak{g}\right)$ is also a Lie supergroup. Let $\mathcal{D}\left(G_{\varepsilon}\right)$ be the convolution algebra of test functions (i.e., smooth compactly supported complex-valued functions) on $G_{\varepsilon}$. The convolution on $\mathcal{D}\left(G_{\varepsilon}\right)$ is defined by

$$
\left(f_{1} \star f_{2}\right)\left(g^{\prime}\right):=\int_{G_{\varepsilon}} f_{1}(g) f_{2}\left(g^{-1} g^{\prime}\right) d g,
$$

where $d g$ is the left-invariant Haar measure. The $*$-algebra structure is given by the involution

$$
\breve{f}(g):=\Delta(g)^{-1} \overline{f\left(g^{-1}\right)},
$$


where $g \mapsto \Delta(g)$ is the modular function satisfying $d\left(g g^{\prime}\right)=\Delta\left(g^{\prime}\right) d g$. From now on, we set

$$
\mathrm{L}_{g} f\left(g^{\prime}\right):=f\left(g^{-1} g^{\prime}\right) \quad \text { and } \quad \mathrm{R}_{x} f(g):=\lim _{t \rightarrow 0} \frac{1}{t}\left(\mathrm{~L}_{e^{t x}} f(g)-f(g)\right),
$$

for $x \in \mathfrak{g}_{0}, g, g^{\prime} \in G_{\boldsymbol{\varepsilon}}, f \in \mathcal{D}\left(G_{\boldsymbol{\varepsilon}}\right)$, and $t \in \mathbb{R}$.

Set $\mathfrak{g}_{\mathbb{C}}:=\mathfrak{g} \otimes_{\mathbb{R}} \mathbb{C}$. For every $g \in G_{\boldsymbol{\varepsilon}}$, let $\alpha_{g}: \boldsymbol{U}\left(\mathfrak{g}_{\mathbb{C}}\right) \rightarrow \boldsymbol{U}\left(\mathfrak{g}_{\mathbb{C}}\right)$ denote the automorphism that is canonically induced by $\operatorname{Ad}(g): \mathfrak{g} \rightarrow \mathfrak{g}$. Our next goal is to define a crossed product $*$-algebra $\mathcal{A}^{\circ}=\mathcal{A}^{\circ}(G, \mathfrak{g})$. As a complex vector space,

$$
\mathcal{A}^{\circ}:=\boldsymbol{U}\left(\mathfrak{g}_{\mathbb{C}}\right) \otimes \mathcal{D}\left(G_{\varepsilon}\right) .
$$

We identify $\mathcal{A}^{\circ}$ with a subspace of the vector space of $\boldsymbol{U}\left(\mathfrak{g}_{\mathbb{C}}\right)$-valued functions on $G_{\varepsilon}$ in the canonical way. Using this identification, we define a multiplication and a complex conjugation on $\mathcal{A}^{\circ}$ by the relations

$$
\left(D_{1} \otimes f_{1}\right)\left(D_{2} \otimes f_{2}\right)\left(g^{\prime}\right):=\int_{G_{\varepsilon}} f_{1}(g) f_{2}\left(g^{-1} g^{\prime}\right) D_{1} \alpha_{g}\left(D_{2}\right) d g
$$

and

$$
(D \otimes f)^{*}(g):=\Delta\left(g^{-1}\right) \overline{f\left(g^{-1}\right)} \alpha_{g}\left(D^{\dagger}\right),
$$

where the map $x \mapsto x^{\dagger}$ is the antilinear antiautomorphism of $\boldsymbol{U}\left(\mathfrak{g}_{\mathbb{C}}\right)$ uniquely defined by

$$
x^{\dagger}:= \begin{cases}-x & \text { if } x \in \mathfrak{g}_{\overline{0}}, \\ -i x & \text { if } x \in \mathfrak{g}_{\overline{1}}\end{cases}
$$

In particular,

$$
\left(D_{1} \otimes f_{1}\right)\left(1 \otimes f_{2}\right)=D_{1} \otimes\left(f_{1} \star f_{2}\right) .
$$

Every $g \in G_{\varepsilon}$ yields a multiplier $\left(\lambda_{g}, \rho_{g}\right)$ of $\mathcal{A}^{\circ}$ by setting

$$
\lambda_{g}(D \otimes f):=\alpha_{g}(D) \otimes \mathrm{L}_{g} f \quad \text { and } \quad \rho_{g}(D \otimes f):=D \otimes \Delta\left(g^{-1}\right) \mathrm{R}_{g^{-1}} f,
$$

where $\mathrm{R}_{g} f\left(g^{\prime}\right):=f\left(g^{\prime} g\right)$. The algebra $\mathcal{A}^{\circ}$ is not necessarily unital. Nevertheless, we have the following lemma.

Lemma 3.1. Every $a \in \mathcal{A}^{\circ}$ can be written as a finite sum $a=a_{1} b_{1}+\cdots+a_{m} b_{m}$, where $a_{k}, b_{k} \in \mathcal{A}^{\circ}$ for $1 \leq k \leq m$. In other words, $\mathcal{A}^{\circ}=\mathcal{A}^{\circ} \mathcal{A}^{\circ}$.

Proof. This follows from the more general result of [Alldridge 2014, Proposition 2.15], but we give a direct and simple argument. It is enough to prove the statement for $a=D \otimes f \in \mathcal{A}^{\circ}$. By the Dixmier-Malliavin theorem [1978], we can write $f=f_{1} \star h_{1}+\cdots+f_{m} \star h_{m}$, where $f_{1}, \ldots, f_{m}, h_{1}, \ldots, h_{m} \in \mathcal{D}\left(G_{\varepsilon}\right)$. It follows that $D \otimes f=\left(D \otimes f_{1}\right)\left(1 \otimes h_{1}\right)+\cdots+\left(D \otimes f_{m}\right)\left(1 \otimes h_{m}\right)$. 
Every unitary representation $\left(\pi, \rho^{\pi}, \mathscr{H}\right)$ of $(G, \mathfrak{g})$ extends to a unitary representation of $\left(G_{\varepsilon}, \mathfrak{g}\right)$ by setting $\pi(\varepsilon) v=(-1)^{|v|} v$ for every homogeneous $v \in \mathscr{H}$. From now on, we assume that every unitary representation of $(G, \mathfrak{g})$ has been extended to $\left(G_{\boldsymbol{\varepsilon}}, \mathfrak{g}\right)$ as indicated above.

Fix a unitary representation $\left(\pi, \rho^{\pi}, \mathscr{H}\right)$ of $(G, \mathfrak{g})$. Let $D \otimes f \in \mathcal{A}^{\circ}$, and as usual set

$$
\pi(f):=\int_{G_{\varepsilon}} f(g) \pi(g) d g .
$$

Note that $\|\pi(f)\| \leq\|f\|_{L^{1}}$. By Gårding's theorem we know that $\pi(f) \mathscr{H} \subseteq \mathscr{H}^{\infty}$, so that the linear map

$$
\rho^{\pi}(D) \pi(f): \mathscr{H} \rightarrow \mathscr{H}
$$

is well-defined.

Proposition 3.2. Let $D \otimes f \in \mathcal{A}^{\circ}$. There exists a constant $M_{D \otimes f}>0$ such that

$$
\left\|\rho^{\pi}(D) \pi(f)\right\| \leq M_{D \otimes f}
$$

for every unitary representation $\left(\pi, \rho^{\pi}, \mathscr{H}\right)$ of $(G, \mathfrak{g})$.

Proof. Note that $\pi(g) \pi(f)=\pi\left(\mathrm{L}_{g} f\right)$ for $g \in G_{\varepsilon}$ and $f \in \mathcal{D}\left(G_{\varepsilon}\right)$ and that for $x \in \mathfrak{g}_{0}$ we have $\lim _{t \rightarrow 0}\left\|\frac{1}{t}\left(\mathrm{~L}_{e^{t x}} f-f\right)-\mathrm{R}_{x} f\right\|_{L^{1}}=0$. Thus, for every $v \in \mathscr{H}$, we obtain that

$$
\begin{aligned}
\mathrm{d} \pi(x) \pi(f) v & =\lim _{t \rightarrow 0} \frac{1}{t}\left(\pi\left(e^{t x}\right) \pi(f) v-\pi(f) v\right) \\
& =\lim _{t \rightarrow 0} \frac{1}{t}\left(\pi\left(\mathrm{L}_{e^{t x}} f\right) v-\pi(f) v\right)=\pi\left(\mathrm{R}_{x} f\right) v .
\end{aligned}
$$

By induction, from (7) it follows that

(8) $\mathrm{d} \pi(D) \pi(f) v=\pi\left(\mathrm{R}_{D} f\right) v \quad$ for $D \in \boldsymbol{U}\left(\mathfrak{g}_{0}\right), f \in \mathcal{D}\left(G_{\boldsymbol{\varepsilon}}\right)$, and $v \in \mathscr{H}$.

If $x \in \mathfrak{g}_{\overline{1}}$, then from (7) it also follows that

$$
\begin{aligned}
\left\|\rho^{\pi}(x) \pi(f) v\right\|^{2} & =\left\langle\rho^{\pi}(x) \pi(f) v, \rho^{\pi}(x) \pi(f) v\right\rangle \\
& =\frac{1}{2}\left|\left\langle\rho^{\pi}([x, x]) \pi(f) v, \pi(f) v\right\rangle\right| \\
& \leq \frac{1}{2}\left\|\rho^{\pi}([x, x]) \pi(f) v\right\| \cdot\|\pi(f) v\| \\
& \leq \frac{1}{2}\left\|\mathrm{R}_{[x, x]} f\right\|_{L^{1}} \cdot\|f\|_{L^{1}} \cdot\|v\|^{2} .
\end{aligned}
$$

Similarly, if $x_{1}, \ldots, x_{d} \in \mathfrak{g}_{1}$ for some $d>1$, then

$$
\begin{aligned}
& \left\|\rho^{\pi}\left(x_{1}\right) \cdots \rho^{\pi}\left(x_{d}\right) \pi(f) v\right\|^{2} \\
& \quad=\left\langle\rho^{\pi}\left(x_{1}\right) \cdots \rho^{\pi}\left(x_{d}\right) \pi(f) v, \rho^{\pi}\left(x_{1}\right) \cdots \rho^{\pi}\left(x_{d}\right) \pi(f) v\right\rangle \\
& \quad \leq \frac{1}{2}\left\|\rho^{\pi}\left(x_{2}\right) \cdots \rho^{\pi}\left(x_{d}\right) \pi(f) v\right\| \cdot\left\|\rho^{\pi}\left(\left[x_{1}, x_{1}\right]\right) \rho^{\pi}\left(x_{2}\right) \cdots \rho^{\pi}\left(x_{d}\right) \pi(f) v\right\| .
\end{aligned}
$$


Furthermore,

$$
\begin{aligned}
& \rho^{\pi}\left(\left[x_{1}, x_{1}\right]\right) \rho^{\pi}\left(x_{2}\right) \cdots \rho^{\pi}\left(x_{d}\right) \pi(f) v \\
& \left.=\sum_{j=2}^{d} \rho^{\pi}\left(x_{2}\right) \cdots \rho^{\pi}\left(\left[x_{1}, x_{1}\right], x_{j}\right]\right) \cdots \rho^{\pi}\left(x_{d}\right) \pi(f) v \\
& \quad+\rho^{\pi}\left(x_{2}\right) \cdots \rho^{\pi}\left(x_{d}\right) \pi\left(\mathrm{R}_{\left[x_{1}, x_{1}\right]} f\right) v .
\end{aligned}
$$

By the Poincaré-Birkhoff-Witt theorem, it is enough to prove the statement of the proposition when

$$
D=y_{1} \cdots y_{\ell^{\prime}} x_{1} \cdots x_{\ell},
$$

where $x_{1}, \ldots, x_{\ell} \in \mathfrak{g}_{\overline{0}}$ and $y_{1}, \ldots, y_{\ell^{\prime}} \in \mathfrak{g}_{\overline{1}}$. From (8), (9), (10), and (11), and by induction on $\ell^{\prime}$, it follows that $\left\|\rho^{\pi}(D) \pi(f)\right\|$ is bounded above by a constant which is expressible in terms of the $L^{1}$-norms of derivatives of $f$.

Remark 3.3. The crossed product algebra $\mathcal{A}^{\circ}$ is also considered in [Alldridge 2014] and [Alldridge et al. 2013]. Here we have introduced two new gadgets: the involution $x \mapsto x^{\dagger}$, and the extension by $\varepsilon$ which we will use below to keep track of the $\mathbb{Z} / 2 \mathbb{Z}$-grading of representations. The $C^{*}$-algebra $\mathcal{A}:=\mathcal{A}(G, \mathfrak{g})$, which will be considered in Section 4, is closely related but not identical to the Fréchet algebra $|\Omega|_{c}(G)$ defined in [Alldridge 2014]. The reader should note that Lemma 4.1 and the results of Section 6 are analogous to the results of Section 2 and, in particular, Proposition 2.15 of that reference. However, our results cannot be obtained as direct consequences of Alldridge's, because a few technical issues arise that one needs to circumvent. In order to address these technical issues, and for the reader's convenience, we provide detailed proofs.

\section{The $C^{*}$-algebra $\mathcal{A}:=\mathcal{A}(G, \mathfrak{g})$}

For a given unitary representation $\left(\pi, \rho^{\pi}, \mathscr{H}\right)$ of $(G, \mathfrak{g})$, we define the linear map $\hat{\pi}: \mathcal{A}^{\circ} \rightarrow B(\mathscr{H})$ by setting

$$
\hat{\pi}(D \otimes f):=\rho^{\pi}(D) \pi(f) \text { for every } D \otimes f \in \mathcal{A}^{\circ},
$$

and then extending $\hat{\pi}$ to $\mathcal{A}^{\circ}$ by linearity. Consider the seminorm on $\mathcal{A}^{\circ}$ defined by

$$
\|a\|:=\sup _{\left(\pi, \rho^{\pi}, \mathscr{H}\right)}\|\hat{\pi}(a)\|,
$$

where the supremum is taken over all unitary equivalence classes of cyclic unitary representations $\left(\pi, \rho^{\pi}, \mathscr{H}\right)$ of $(G, \mathfrak{g})$. From Proposition 3.2 it follows that $\|a\|<\infty$.

Lemma 4.1. $\hat{\pi}$ is a $*$-representation of $\mathcal{A}^{\circ}$.

Proof. This is the analogue of [Alldridge 2014, Lemma 2.14]. First we prove that $\hat{\pi}(a b)=\hat{\pi}(a) \hat{\pi}(b)$ for every $a, b \in \mathcal{A}^{\circ}$. It is enough to assume that $a=$ 
$D_{1} \otimes f_{1}$ and $b=D_{2} \otimes f_{2}$. Choose $\eta_{1}, \ldots, \eta_{r} \in \mathcal{C}^{\infty}\left(G_{\varepsilon}\right)$ and $E_{1}, \ldots, E_{r} \in \boldsymbol{U}\left(\mathfrak{g}_{\mathbb{C}}\right)$ such that $\alpha_{g}\left(D_{2}\right)=\sum_{i=1}^{r} \eta_{i}(g) E_{i}$. Fix $v \in \mathscr{H}$ and set $w:=\pi\left(f_{2}\right) v$. Then $w \in$ $\mathscr{H}^{\infty}$ and therefore the map $G_{\varepsilon} \rightarrow \mathscr{H}^{\infty}, g \mapsto \pi(g) w$ is smooth [Poulsen 1972, Proposition 2.1]. Using Proposition 2.6 we obtain

$$
\begin{aligned}
\hat{\pi}(a) \hat{\pi}(b) v & =\rho^{\pi}\left(D_{1}\right) \int_{G_{\varepsilon}} f_{1}(g) \pi(g) \rho^{\pi}\left(D_{2}\right) \pi\left(f_{2}\right) v d g \\
& =\rho^{\pi}\left(D_{1}\right) \sum_{i=1}^{r} \rho^{\pi}\left(E_{i}\right) \pi\left(\eta_{i} f_{1}\right) \pi\left(f_{2}\right) v \\
& =\hat{\pi}\left(\sum_{i=1}^{r} D_{1} E_{i} \otimes\left(\eta_{i} f_{1} * f_{2}\right)\right) v \\
& =\hat{\pi}(a b) v .
\end{aligned}
$$

The equality $\hat{\pi}(a)^{*}=\hat{\pi}\left(a^{*}\right)$ can be verified by a similar calculation, using the relation

$$
\left\langle\rho^{\pi}(D \otimes f) v, w\right\rangle=\left\langle\rho^{\pi}(D) \pi(f) v, w\right\rangle=\left\langle v, \pi(\breve{f}) \rho^{\pi}\left(D^{\dagger}\right) w\right\rangle,
$$

where $\breve{f}(g)=\Delta(g)^{-1} \overline{f\left(g^{-1}\right)}$.

We are now ready to define $\mathcal{A}:=\mathcal{A}(G, \mathfrak{g})$. From Lemma 4.1 it follows that the map $a \mapsto a^{*}$ is an isometry of $\mathcal{A}^{\circ}$ and that $\left\|a a^{*}\right\|=\|a\|^{2}$. Set $\mathcal{A}_{-}^{\circ}:=\left\{a \in \mathcal{A}^{\circ}:\|a\|=0\right\}$ and let $\mathcal{A}$ denote the completion of the quotient $\mathcal{A}^{\circ} / \mathcal{A}_{-}^{\circ}$ with respect to its induced norm. It is straightforward to check that $\mathcal{A}$ is a $C^{*}$-algebra.

Lemma 4.2. Let $f \in \mathcal{D}\left(G_{\varepsilon}\right)$ and $D \otimes h \in \mathcal{A}^{\circ}$. Then the map

$$
\gamma_{f, D, h}: G_{\varepsilon} \rightarrow \mathcal{A}, \quad g \mapsto f(g) \alpha_{g}(D) \otimes \mathrm{L}_{g} h
$$

is continuous and

$$
\int_{G_{\varepsilon}} \gamma_{f, D, h}(g) d g=(1 \otimes f)(D \otimes h) .
$$

Proof. Choose $E_{1}, \ldots, E_{r} \in \boldsymbol{U}\left(\mathfrak{g}_{\mathbb{C}}\right)$ and $\eta_{1}, \ldots, \eta_{r} \in \mathcal{C}^{\infty}\left(G_{\boldsymbol{\varepsilon}}\right)$ such that we have $\alpha_{g}(D)=\sum_{i=1}^{r} \eta_{i}(g) E_{i}$ for every $g \in G_{\boldsymbol{\varepsilon}}$. Then $\gamma_{f, D, h}(g)=\sum_{i=1}^{r} E_{i} \otimes f(g) \eta_{i}(g) \mathrm{L}_{g} h$. Next we prove that, for every $1 \leq i \leq r$, the map $G_{\varepsilon} \rightarrow \mathcal{A}, g \mapsto E_{i} \otimes \mathrm{L}_{g} h$ is continuous. Since we can replace $h$ by $\mathrm{L}_{g} h$, it suffices to prove continuity at $\mathbf{1} \in G_{\varepsilon}$. To this end, we need to show that

$$
\lim _{g \rightarrow \mathbf{1}}\left(\sup _{\left(\pi, \rho^{\pi}, \mathscr{H}\right)}\left\|\rho^{\pi}\left(E_{i}\right) \pi\left(\mathrm{L}_{g} h-h\right)\right\|\right)=0
$$


By an argument similar to the proof of Proposition 3.2, the latter statement can be reduced to showing that $\lim _{g \rightarrow \mathbf{1}}\left\|\mathrm{R}_{D}\left(\mathrm{~L}_{g} h-h\right)\right\|_{L^{1}}=0$ for every $D \in \boldsymbol{U}\left(\mathfrak{g}_{\overline{0}}\right)$. This is straightforward.

Next we prove (14). From (3) it follows that

$$
\begin{aligned}
(1 \otimes f)(D \otimes h)\left(g^{\prime}\right) & =\sum_{i=1}^{r} \int_{G_{\varepsilon}} f(g)\left(\mathrm{L}_{g} h\right)\left(g^{\prime}\right) \eta_{i}(g) E_{i} d g \\
& =\sum_{i=1}^{r}\left(f \eta_{i} \star h\right)\left(g^{\prime}\right) E_{i} .
\end{aligned}
$$

The left-regular representation of $G_{\varepsilon}$ on $L^{1}\left(G_{\varepsilon}\right)$ is strongly continuous, and its integrated representation is given by convolution, that is, $\int_{G_{\varepsilon}} f(g) \mathrm{L}_{g} h d g=f \star h$ for every $f, h \in L^{1}\left(G_{\varepsilon}\right)$. We can now finish the proof by an argument similar to the one for [Alldridge 2014, (2.13)]. More precisely, we have

$$
\int_{G_{\varepsilon}} \gamma_{f, D, h}(g) d g=\sum_{i=1}^{r} E_{i} \otimes \int_{G_{\varepsilon}} f(g) \eta_{i}(g) \mathrm{L}_{g} h d g=\sum_{i=1}^{r} E_{i} \otimes\left(\left(f \eta_{i}\right) \star h\right) .
$$

Equality (14) now follows from (15) and (16).

\section{Multipliers of $\mathcal{A}$ and $\mathcal{A}^{\circ}$}

For every $g \in G_{\varepsilon}$, let $\left(\lambda_{g}, \rho_{g}\right)$ be the multiplier of $\mathcal{A}^{\circ}$ that is defined in (6). It is straightforward to verify that $\lambda_{g}$ and $\rho_{g}$ are isometries of $\mathcal{A}^{\circ}$, and therefore the multiplier $\left(\lambda_{g}, \rho_{g}\right)$ extends uniquely to a multiplier of $\mathcal{A}$. For every $g \in G_{\boldsymbol{\varepsilon}}$, the map

$$
\eta_{G}(g): \mathcal{A} \rightarrow \mathcal{A}, \quad a \mapsto \lambda_{g}(a)
$$

is an isometry and we have $\eta_{G}\left(g g^{\prime}\right)=\eta_{G}(g) \eta_{G}\left(g^{\prime}\right)$.

Proposition 5.1. For every $a \in \mathcal{A}^{\circ}$, the map

$$
G \rightarrow \mathcal{A}, \quad g \mapsto \eta_{G}(g) a
$$

is smooth.

Proof. It suffices to prove that the orbit map of every $D \otimes f \in \mathcal{A}^{\circ}$ is smooth. Set

$$
\hat{\pi}_{u}:=\bigoplus_{\left(\pi, \rho^{\pi}, \mathscr{H}\right)} \hat{\pi} \quad \text { and } \quad\left(\pi_{u}, \mathscr{H}_{u}\right):=\bigoplus_{\left(\pi, \rho^{\pi}, \mathscr{H}\right)}(\pi, \mathscr{H})
$$

where the direct sums are over unitary equivalence classes of cyclic unitary representations of $(G, \mathfrak{g})$. Then $\left(\pi_{u}, \mathscr{H}_{u}\right)$ is a smooth unitary representation of $G_{\boldsymbol{\varepsilon}}$, and the map $\hat{\pi}_{u}: \mathcal{A}^{\circ} \rightarrow B\left(\mathscr{H}_{u}\right)$ is an isometry. Furthermore, $\hat{\pi}_{u}\left(\eta_{G}(g) a\right)=\pi_{u}(g) \hat{\pi}_{u}(a)$ 
for $g \in G_{\varepsilon}$ and $a \in \mathcal{A}^{\circ}$. Consequently, to complete the proof it suffices to show that, for every $a \in \mathcal{A}^{\circ}$, the map

$$
G \rightarrow B\left(\mathscr{H}_{u}\right), \quad g \mapsto \pi_{u}(g) \hat{\pi}_{u}(a)
$$

is smooth. The latter statement is a consequence of [Neeb et al. 2015, Theorem 2.11]. Indeed, by the same theorem we need to verify that $\hat{\pi}_{u}(a) \mathscr{H} \subseteq \mathscr{H}^{\infty}$. Without loss of generality we can assume $a=D \otimes f$, and therefore $\hat{\pi}_{u}(a)=\rho^{\pi_{u}}(D) \pi_{u}(f)$. From Gårding's theorem and Definition 2.3(ii), we obtain $\hat{\pi}_{u}(a) \mathscr{H} \subseteq \mathscr{H}^{\infty}$.

By [Fell and Doran 1988b, Propositions VIII.1.11 and VIII.1.18], every multiplier of $\mathcal{A}$ is bounded and the multipliers of $\mathcal{A}$ form a unital Banach $*$-algebra $M(\mathcal{A})$ with multiplication and complex conjugation defined in (2) and the norm defined by $\|(\lambda, \rho)\|:=\max \{\|\lambda\|,\|\rho\|\}$. Furthermore, the multipliers $\left(\lambda_{g}, \rho_{g}\right)$ for $g \in G_{\varepsilon}$ are unitary, that is,

$$
\left(\lambda_{g}, \rho_{g}\right)\left(\lambda_{g}, \rho_{g}\right)^{*}=1 \in M(\mathcal{A}) .
$$

\section{Nondegenerate $*$-representations of $\mathcal{A}$}

In this section we prove that the category of unitary representations of $(G, \mathfrak{g})$ is isomorphic to the category of nondegenerate (in the sense of [Fell and Doran 1988a, Definition V.1.7]) $*$-representations of the $C^{*}$-algebra $\mathcal{A}=\mathcal{A}(G, \mathfrak{g})$.

Proposition 6.1. Let $\left(\pi, \rho^{\pi}, \mathscr{H}\right)$ be a unitary representation of a Lie supergroup $(G, \mathfrak{g})$. Then the $*$-representation $\hat{\pi}$ defined in Lemma 4.1 extends in a unique way to a nondegenerate $*$-representation

$$
\hat{\pi}: \mathcal{A} \rightarrow B(\mathscr{H}) .
$$

Proof. From (13) and Remark 2.5 it follows that $\|\hat{\pi}(a)\| \leq\|a\|$ for every $a \in \mathcal{A}^{\circ}$. The existence and uniqueness of the extension $\hat{\pi}: \mathcal{A} \rightarrow B(\mathscr{H})$ now follows immediately. Nondegeneracy of $\hat{\pi}$ follows from the equality $\hat{\pi}(1 \otimes f)=\pi(f)$ for $f \in \mathcal{D}\left(G_{\varepsilon}\right)$.

We now give a construction of a unitary representation of $(G, \mathfrak{g})$ from a nondegenerate $*$-representation $\hat{\pi}: \mathcal{A} \rightarrow B(\mathscr{H})$ of $\mathcal{A}$. By [Fell and Doran 1988b, Propositions VIII.1.11 and VIII.1.12], there exists a unique extension of $\hat{\pi}$ to a *-representation $\hat{\pi}: M(\mathcal{A}) \rightarrow B(\mathscr{H})$ of the multiplier algebra $M(\mathcal{A})$ satisfying

$$
\hat{\pi}((\lambda, \rho)) \hat{\pi}(a)=\hat{\pi}(\lambda(a)) \quad \text { for }(\lambda, \rho) \in M(\mathcal{A}) \text { and } a \in \mathcal{A} .
$$

Set

$$
\pi(g):=\hat{\pi}\left(\left(\lambda_{g}, \rho_{g}\right)\right) \text { for every } g \in G_{\varepsilon} .
$$

From (18) it follows that

$$
\pi\left(g^{-1}\right)=\hat{\pi}\left(\left(\lambda_{g^{-1}}, \rho_{g^{-1}}\right)\right)=\hat{\pi}\left(\left(\lambda_{g}, \rho_{g}\right)^{*}\right)=\hat{\pi}\left(\left(\lambda_{g}, \rho_{g}\right)\right)^{*}=\pi(g)^{*},
$$


that is, the operators $\pi(g)$ are unitary. Furthermore, from (19) it follows that the subspace $\mathscr{H}^{\circ}:=\hat{\pi}\left(\mathcal{A}^{\circ}\right) \mathscr{H}$ is invariant under $\pi(g)$ for every $g \in G_{\varepsilon}$.

Lemma 6.2. For every $v \in \mathscr{H}$, the map $G_{\varepsilon} \rightarrow \mathscr{H}, g \mapsto \pi(g) v$ is smooth if and only if $v \in \mathscr{H}^{\circ}$. In particular, $(\pi, \mathscr{H})$ is a smooth unitary representation of $G$.

Proof. The statement and proof are analogous to [Alldridge 2014, Proposition 2.15], but there are subtle technical differences, and therefore we provide a detailed argument. First we show that, for every $v \in \mathscr{H}^{\circ}$, the orbit map $G_{\varepsilon} \rightarrow \mathscr{H}, g \mapsto \pi(g) v$ is smooth. Assume that $v=\hat{\pi}(a) w$ for $a \in \mathcal{A}^{\circ}$ and $w \in \mathscr{H}$. Then

$$
\pi(g) v=\hat{\pi}\left(\eta_{G}(g) a\right) w,
$$

where $\eta_{G}(g): \mathcal{A} \rightarrow \mathcal{A}$ is defined in (17). Since the map $\mathcal{A} \rightarrow \mathscr{H}, a \mapsto \hat{\pi}(a) w$ is continuous and linear, Proposition 5.1 implies that the orbit map $g \mapsto \pi(g) v$ is smooth.

Next we observe that $\mathscr{H}^{\circ}$ is a dense subspace of $\mathscr{H}$, because $\mathcal{A}^{\circ}$ is a dense subspace of $\mathcal{A}$. Therefore, the representation $(\pi, \mathscr{H})$ is smooth.

Finally, we prove that every smooth vector of $(\pi, \mathscr{H})$ belongs to $\mathscr{H}^{\circ}$. By the Dixmier-Malliavin theorem, it is enough to show that

$$
\pi(f)=\hat{\pi}(1 \otimes f) \text { for every } f \in \mathcal{D}\left(G_{\varepsilon}\right),
$$

where $\pi(f) v:=\int_{G_{\varepsilon}} f(g) \pi(g) v d g$ for $v \in \mathscr{H}$. Since both sides of (21) are bounded operators and $\mathscr{H}^{\circ}$ is dense in $\mathscr{H}$, it is enough to prove that

$$
\pi(f) \hat{\pi}(D \otimes h) v=\hat{\pi}((1 \otimes f)(D \otimes h)) v \quad \text { for } D \otimes h \in \mathcal{A}^{\circ} \text { and } v \in \mathscr{H} .
$$

Let $\gamma_{f, D, h}$ be defined as in Lemma 4.2. From (20) and (19) it follows that, for every $v \in \mathscr{H}$,

$$
\begin{aligned}
\pi(f) \hat{\pi}(D \otimes h) v & =\int_{G_{\varepsilon}} f(g) \pi(g) \hat{\pi}(D \otimes h) v d g \\
& =\int_{G_{\varepsilon}} \hat{\pi}\left(\gamma_{f, D, h}(g)\right) v d g=\hat{\pi}\left(\int_{G_{\varepsilon}} \gamma_{f, D, h}(g) d g\right) v,
\end{aligned}
$$

and from (14) it follows that $\pi(f) \hat{\pi}(D \otimes h) v=\hat{\pi}((1 \otimes f)(D \otimes h)) v$.

Set

$$
\pi^{\circ}(a):=\left.\hat{\pi}(a)\right|_{\mathscr{H}} \circ \quad \text { for every } a \in \mathcal{A}^{\circ} .
$$

From Lemmas 3.1 and 6.2 it follows that $\left(\pi^{\circ}, \mathscr{H}^{\circ}\right)$ is a nondegenerate $*$-representation of $\mathcal{A}^{\circ}$ in the sense defined in [Fell and Doran 1988a, Definition IV.3.17]. Therefore, by [Fell and Doran 1988b, Proposition VIII.1.9] there exists a unique extension of $\pi^{\circ}$ to a $*$-representation $\pi^{\circ}: M\left(\mathcal{A}^{\circ}\right) \rightarrow \operatorname{End}_{\mathbb{C}}\left(\mathscr{H}^{\circ}\right)$ satisfying

$$
\pi^{\circ}((\lambda, \rho)) \pi^{\circ}(a)=\pi^{\circ}(\lambda(a)) \text { for }(\lambda, \rho) \in M\left(\mathcal{A}^{\circ}\right) \text { and } a \in \mathcal{A}^{\circ} .
$$


From the latter equality, Lemma 3.1, and (19), it follows that $\pi^{\circ}\left(\left(\lambda_{g}, \rho_{g}\right)\right)=$ $\left.\pi(g)\right|_{\mathscr{H}} \circ$ for every $g \in G_{\varepsilon}$.

For every $x \in \mathfrak{g}$, let $\left(\lambda_{x}, \rho_{x}\right) \in M\left(\mathcal{A}^{\circ}\right)$ be the multiplier defined by

$$
\lambda_{x}(D \otimes f):=x D \otimes f \quad \text { and } \quad \rho_{x}(D \otimes f)(g):=f(g) D \alpha_{g}(x) \quad \text { for } g \in G_{\boldsymbol{\varepsilon}} .
$$

It is straightforward to verify that $\left(\lambda_{x}, \rho_{x}\right)^{*}=\left(\lambda_{x^{\dagger}}, \rho_{x^{\dagger}}\right)$ for every $x \in \mathfrak{g}$, where $x^{\dagger}$ is defined as in (5). For every $x \in \mathfrak{g}$, we define a linear map

$$
\rho^{\pi}(x): \mathscr{H}^{\circ} \rightarrow \mathscr{H}^{\circ}, \quad v \mapsto \pi^{\circ}\left(\left(\lambda_{x}, \rho_{x}\right)\right) v .
$$

Since $\pi(\boldsymbol{\varepsilon})^{2}=\mathbf{1}$, we obtain a $\mathbb{Z} / 2 \mathbb{Z}$-grading $\mathscr{H}=\mathscr{H}_{0} \oplus \mathscr{H}_{1}$ by the \pm 1 eigenspaces of $\pi(\varepsilon)$, i.e.,

$$
\mathscr{H}_{0}:=\{v \in \mathscr{H}: \pi(\varepsilon) v=v\} \quad \text { and } \quad \mathscr{H}_{1}:=\{v \in \mathscr{H}: \pi(\varepsilon) v=-v\} .
$$

Since $\pi(\varepsilon)$ leaves $\mathscr{H}^{\circ}$ invariant, the $\mathbb{Z} / 2 \mathbb{Z}$-grading of $\mathscr{H}$ induces a $\mathbb{Z} / 2 \mathbb{Z}$-grading $\mathscr{H}^{\circ}=\mathscr{H}_{\overline{0}}^{\circ} \oplus \mathscr{H}_{\overline{1}}^{\circ}$ on $\mathscr{H}^{\circ}$. We now prove the following proposition.

Proposition 6.3. $\left(\pi, \rho^{\pi}, \mathscr{H}\right)$ is a unitary representation of $(G, \mathfrak{g})$.

Proof. Every $g \in G$ commutes with $\boldsymbol{\varepsilon}$, and therefore $\pi(g)$ preserves the $\mathbb{Z} / 2 \mathbb{Z}$-grading of $\mathscr{H}$. If $x \in \mathfrak{g}_{\overline{1}}$, then $\left(\lambda_{x}, \rho_{x}\right)^{*}=\left(\rho_{-i x}, \lambda_{-i x}\right)$ in $M\left(\mathcal{A}^{\circ}\right)$, and it follows that the operator $e^{-\pi i / 4} \rho^{\pi}(x)$ is symmetric. For every $x \in \mathfrak{g}$ and $g \in G_{\varepsilon}$, we have

$$
\left(\lambda_{g}, \rho_{g}\right)\left(\lambda_{x}, \rho_{x}\right)\left(\lambda_{g^{-1}}, \rho_{g^{-1}}\right)=\left(\lambda_{\operatorname{Ad}(g) x}, \rho_{\operatorname{Ad}(g) x}\right),
$$

and consequently

$$
\pi(g) \rho^{\pi}(x) \pi(g)^{-1}=\rho^{\pi}(\operatorname{Ad}(g) x) .
$$

In particular, from (23) for $g=\boldsymbol{\varepsilon}$, it follows that $\rho^{\pi}(x) \in \operatorname{End}_{\mathbb{C}}\left(\mathscr{H}^{\circ}\right)_{\overline{0}}$ for $x \in \mathfrak{g}_{\overline{0}}$ and $\rho^{\pi}(x) \in \operatorname{End}_{\mathbb{C}}\left(\mathscr{H}^{\circ}\right)_{\overline{1}}$ for $x \in \mathfrak{g}_{\overline{1}}$. The relation $\rho^{\pi}([x, y])=\left[\rho^{\pi}(x), \rho^{\pi}(y)\right]$ for $x, y \in \mathfrak{g}$ follows from the corresponding relation in the multiplier algebra $M\left(\mathcal{A}^{\circ}\right)$. Finally, we prove that $\rho^{\pi}(x)=\left.\mathrm{d} \pi(x)\right|_{\mathscr{H}}$ for every $x \in \mathfrak{g}_{0}$. Fix $a \in \mathcal{A}^{\circ}$ and $v \in \mathscr{H}^{\circ}$, and set

$$
\phi_{a, t}:=\frac{1}{t}\left(\pi\left(e^{t x}\right) \pi^{\circ}(a) v-\pi^{\circ}(a) v\right)-\rho^{\pi}(x) \pi^{\circ}(a) v \in \mathscr{H} .
$$

Then $\phi_{a, t}=\pi^{\circ}\left(a_{t}\right) v$, where $a_{t}:=\frac{1}{t}\left(\lambda_{e^{t x}}(a)-a\right)-\lambda_{x}(a) \in \mathcal{A}^{\circ}$. To complete the proof, we need to show that $\lim _{t \rightarrow 0}\left\|\phi_{a, t}\right\|=0$. But

$$
\left\|\phi_{a, t}\right\|=\left\|\pi^{\circ}\left(a_{t}\right) v\right\|=\left\|\hat{\pi}\left(a_{t}\right) v\right\| \leq\left\|a_{t}\right\| \cdot\|v\|,
$$

and therefore it suffices to show that $\lim _{t \rightarrow 0}\left\|a_{t}\right\|=0$. Without loss of generality we can assume that $a=D \otimes f$. From the definition of the norm of $\mathcal{A}$ we obtain

$$
\left\|a_{t}\right\|=\sup _{\left(\sigma, \rho^{\sigma}, \mathscr{K}\right)}\left\|\frac{1}{t}\left(\sigma\left(e^{t x}\right) \rho^{\sigma}(D) \sigma(f)-\rho^{\sigma}(D) \sigma(f)\right)-\rho^{\sigma}(x D) \sigma(f)\right\|,
$$


where the supremum is taken over all unitary equivalence classes of cyclic unitary representations $\left(\sigma, \rho^{\sigma}, \mathscr{K}\right)$ of $(G, \mathfrak{g})$. Now fix a unitary representation $\left(\sigma, \rho^{\sigma}, \mathscr{K}\right)$ and a vector $v \in \mathscr{H}_{\sigma}$ such that $\|v\|=1$. By Taylor's theorem,

$$
\begin{aligned}
& \sigma\left(e^{t x}\right) \rho^{\sigma}(D) \sigma(f) v \\
& \quad=\rho^{\sigma}(D) \sigma(f) v+t \rho^{\sigma}(x D) \sigma(f) v+\frac{1}{2} \int_{0}^{t}(t-s) \sigma\left(e^{s x}\right) \rho^{\sigma}\left(x^{2} D\right) \sigma(f) v d s .
\end{aligned}
$$

Proposition 3.2 implies that there exists a constant $M>0$, independent of $\left(\sigma, \rho^{\sigma}, \mathscr{K}\right)$, such that $\left\|\rho^{\sigma}\left(x^{2} D\right) \sigma(f)\right\| \leq M$. It follows that $\left\|a_{t}\right\| \leq \frac{1}{2} M \cdot|t|$, and consequently $\lim _{t \rightarrow 0}\left\|a_{t}\right\|=0$.

Recall that the morphisms in the two categories of unitary representations of $(G, \mathfrak{g})$, and nondegenerate $*$-representations of $\mathcal{A}=\mathcal{A}(G, \mathfrak{g})$, are bounded linear intertwining operators.

Theorem 6.4. The correspondences of Propositions 6.1 and 6.3 result in an isomorphism between the category of unitary representations of $(G, \mathfrak{g})$ and the category of nondegenerate $*$-representations of $\mathcal{A}=\mathcal{A}(G, \mathfrak{g})$.

Proof. Step 1. First we verify that the correspondences of Propositions 6.1 and 6.3 are mutual inverses. Let $\left(\pi, \rho^{\pi}, \mathscr{H}\right)$ be a unitary representation of $(G, \mathfrak{g})$. Let $\hat{\pi}$ be the $*$-representation of $\mathcal{A}$ constructed by Proposition 6.1, and let $\left(\bar{\pi}, \rho^{\bar{\pi}}, \mathscr{H}\right.$ ) be the unitary representation of $(G, \mathfrak{g})$ constructed from $\hat{\pi}$ by Proposition 6.3. For $D \otimes f \in \mathcal{A}^{\circ}$ and $g \in G_{\varepsilon}$,

$$
\begin{aligned}
\bar{\pi}(g) \hat{\pi}(D \otimes f) & =\hat{\pi}\left(\lambda_{g}(D \otimes f)\right)=\hat{\pi}\left(\alpha_{g}(D) \otimes \mathrm{L}_{g} f\right) \\
& =\rho^{\pi}\left(\alpha_{g}(D)\right) \pi\left(\mathrm{L}_{g} f\right)=\pi(g) \rho^{\pi}(D) \pi(f)=\pi(g) \hat{\pi}(D \otimes f) .
\end{aligned}
$$

Since $\pi(g)$ and $\bar{\pi}(g)$ are bounded operators and $\hat{\pi}$ is nondegenerate, we obtain $\pi(g)=\bar{\pi}(g)$ for $g \in G_{\varepsilon}$. Let $\mathscr{H}^{\infty}$ denote the space of smooth vectors of $(\pi, \mathscr{H})$. For $x \in \mathfrak{g}, D \otimes f \in \mathcal{A}^{\circ}$, and $w \in \mathscr{H}$,

$$
\begin{aligned}
\rho^{\bar{\pi}}(x) \hat{\pi}(D \otimes f) w & =\hat{\pi}(x D \otimes f) w=\rho^{\pi}(x D) \pi(f) w \\
& =\rho^{\pi}(x) \rho^{\pi}(D) \pi(f) w=\rho^{\pi}(x) \hat{\pi}(D \otimes f) w .
\end{aligned}
$$

By the Dixmier-Malliavin theorem, $\mathscr{H}^{\infty}=\hat{\pi}\left(\mathcal{A}^{\circ}\right) \mathscr{H}$. Therefore, (24) implies that $\rho^{\bar{\pi}}(x)=\rho^{\pi}(x)$ for every $x \in \mathfrak{g}$.

Conversely, let $\hat{\pi}: \mathcal{A} \rightarrow B(\mathscr{H})$ be a nondegenerate $*$-representation. Let $\left(\pi, \rho^{\pi}, \mathscr{H}\right)$ be the unitary representation of $(G, \mathfrak{g})$ corresponding to $\hat{\pi}$ by Proposition 6.3, and let $\hat{\pi}^{\prime}: \mathcal{A} \rightarrow B(\mathscr{H})$ be the $*$-representation corresponding to $\left(\pi, \rho^{\pi}, \mathscr{H}\right)$ by 
Proposition 6.1. For $D_{1} \otimes h_{1} \in \mathcal{A}^{\circ}$ and $w \in \mathscr{H}$, we obtain by Lemma 4.2 that

$$
\begin{aligned}
\pi(f) \hat{\pi}\left(D_{1} \otimes h_{1}\right) w & =\int_{G_{\varepsilon}} f(g) \pi(g) \hat{\pi}\left(D_{1} \otimes h_{1}\right) w d g \\
& =\int_{G_{\varepsilon}} \hat{\pi}\left(\gamma_{f, D_{1}, h_{1}}(g)\right) w d g \\
& =\hat{\pi}\left(\int_{G_{\varepsilon}} \gamma_{f, D_{1}, h_{1}}(g) d g\right) w \\
& =\hat{\pi}\left((1 \otimes f)\left(D_{1} \otimes h_{1}\right)\right) w .
\end{aligned}
$$

Now set $a:=D_{1} \otimes h_{1}$. From (25) it follows that, for every $D \otimes f \in \mathcal{A}^{\circ}$,

$$
\begin{aligned}
\hat{\pi}^{\prime}(D \otimes f) \hat{\pi}(a) w & =\rho^{\pi}(D) \pi(f) \hat{\pi}(a) w \\
& =\rho^{\pi}(D) \hat{\pi}((1 \otimes f) a) w=\hat{\pi}(D \otimes f) \hat{\pi}(a) w .
\end{aligned}
$$

Nondegeneracy of $\hat{\pi}$ and boundedness of the operators $\hat{\pi}^{\prime}(D \otimes f)$ and $\hat{\pi}(D \otimes f)$ imply that $\hat{\pi}^{\prime}(D \otimes f)=\hat{\pi}(D \otimes f)$. Since $\mathcal{A}^{\circ}$ is dense in $\mathcal{A}$, the equality $\hat{\pi}^{\prime}(a)=\hat{\pi}(a)$ holds for every $a \in \mathcal{A}$.

Step 2. To complete the proof, we need to show that the correspondences of Propositions 6.1 and 6.3 are compatible with morphisms in the two categories. Suppose that $\left(\pi, \rho^{\pi}, \mathscr{H}\right)$ and $\left(\sigma, \rho^{\sigma}, \mathscr{K}\right)$ are two unitary representations of $(G, \mathfrak{g})$, and let $\hat{\pi}: \mathcal{A} \rightarrow B(\mathscr{H})$ and $\hat{\sigma}: \mathcal{A} \rightarrow B(\mathscr{K})$ be the $*$-representations of $\mathcal{A}$ constructed from $\left(\pi, \rho^{\pi}, \mathscr{H}\right)$ and $\left(\sigma, \rho^{\sigma}, \mathscr{K}\right)$ by Proposition 6.1. If $T: \mathscr{H} \rightarrow \mathscr{K}$ is a $(G, \mathfrak{g})$ intertwining operator, then it is easy to verify that $T$ commutes with the action of $\mathcal{A}^{\circ}$ on $\mathscr{H}$ and $\mathscr{K}$, and therefore, by a continuity argument, $T$ commutes with the action of $\mathcal{A}$ on $\mathscr{H}$ and $\mathscr{K}$ as well.

Conversely, assume that $T: \mathscr{H} \rightarrow \mathscr{K}$ commutes with the actions of $\mathcal{A}$ on $\mathscr{H}$ and $\mathscr{K}$. First note that, for every $a \in \mathcal{A}$ and every $(\lambda, \rho) \in M(\mathcal{A})$,

$T \hat{\pi}((\lambda, \rho)) \hat{\pi}(a)=T \hat{\pi}(\lambda(a))=\hat{\sigma}(\lambda(a)) T=\hat{\sigma}((\lambda, \rho)) \hat{\sigma}(a) T=\hat{\sigma}((\lambda, \rho)) T \hat{\pi}(a)$.

Since $\hat{\pi}(\mathcal{A}) \mathscr{H}$ is a dense subspace of $\mathscr{H}$, it follows that

$$
T \hat{\pi}((\lambda, \rho))=\hat{\sigma}((\lambda, \rho)) T \quad \text { for every }(\lambda, \rho) \in M(\mathcal{A}) \text {. }
$$

Setting $(\lambda, \rho):=\left(\lambda_{g}, \rho_{g}\right)$ in the last relation, we obtain

$$
T \pi(g)=\sigma(g) T \quad \text { for every } g \in G_{\varepsilon},
$$

and in particular $T \mathscr{H}^{\infty} \subseteq \mathscr{K}^{\infty}$. From (26) for $g=\varepsilon$, it follows that $T$ preserves the $\mathbb{Z} / 2 \mathbb{Z}$-grading of $\mathscr{H}$. Now for $(\lambda, \rho) \in M\left(\mathcal{A}^{\circ}\right), a \in \mathcal{A}^{\circ}$, and $v \in \mathscr{H}^{\infty}$, using 
Lemma 6.2 we obtain that

$$
\begin{aligned}
T \pi^{\circ}(\lambda, \rho) \pi^{\circ}(a) v & =T \pi^{\circ}(\lambda(a)) v=\sigma^{\circ}(\lambda(a)) T v \\
& =\sigma^{\circ}(\lambda, \rho) \sigma^{\circ}(a) T v=\sigma^{\circ}(\lambda, \rho) T \pi^{\circ}(a) v .
\end{aligned}
$$

Thus Lemmas 6.2 and 3.1 imply that $T \pi^{\circ}(\lambda, \rho) w=\sigma^{\circ}(\lambda, \rho) T w$ for every $w \in \mathscr{H}^{\infty}$. Setting $(\lambda, \rho):=\left(\lambda_{x}, \rho_{x}\right)$ for $x \in \mathfrak{g}$, we obtain $T \rho^{\pi}(x)=\rho^{\sigma}(x) T$. Therefore, $T$ is a $(G, \mathfrak{g})$-intertwining map from $\left(\pi, \rho^{\pi}, \mathscr{H}\right)$ to $\left(\sigma, \rho^{\sigma}, \mathscr{K}\right)$.

\section{Unique direct integral decompositions}

For a unitary representation $\left(\pi, \rho^{\pi}, \mathscr{H}\right)$ of a Lie supergroup $(G, \mathfrak{g})$, it is desirable to have a decomposition as a direct integral of irreducible unitary representations. From Theorem 6.4 it follows that the problem of existence and uniqueness of such a direct integral decomposition can be reduced to the same problem for the associated $C^{*}$-algebra $\mathcal{A}=\mathcal{A}(G, \mathfrak{g})$.

In this section we prove that existence and uniqueness of direct integral decompositions hold for two general classes of Lie supergroups, which include nilpotent and basic classical Lie supergroups.

Recall that a $C^{*}$-algebra $\mathcal{A}$ is called $C C R$ if $\hat{\pi}(\mathcal{A}) \subseteq K(\mathscr{H})$ for every irreducible *-representation $\hat{\pi}: \mathcal{A} \rightarrow B(\mathscr{H})$, where $K(\mathscr{H}) \subseteq B(\mathscr{H})$ denotes the subspace of compact operators. It is well known that, for $C^{*}$-algebras which are CCR, existence and uniqueness of direct integral decompositions hold.

A unitary representation $(\pi, \mathscr{H})$ of a Lie group $G$ is called completely continuous if $\pi(f) \in K(\mathscr{H})$ for every $f \in \mathcal{D}(G)$.

Theorem 7.1. Let $(G, \mathfrak{g})$ be a Lie supergroup such that, for every irreducible unitary representation $\left(\pi, \rho^{\pi}, \mathscr{H}\right)$ of $(G, \mathfrak{g})$, the unitary representation $(\pi, \mathscr{H})$ of $G$ is completely continuous. Then the $C^{*}$-algebra $\mathcal{A}=\mathcal{A}(G, \mathfrak{g})$ is $C C R$.

Proof. Let $\hat{\pi}: \mathcal{A} \rightarrow B(\mathscr{H})$ be an irreducible $*$-representation of $\mathcal{A}$. Since $K(\mathscr{H})$ is a closed ideal of $B(\mathscr{H})$ and $\|\hat{\pi}(a)\| \leq\|a\|$ for every $a \in \mathcal{A}$, it suffices to prove that $\hat{\pi}(D \otimes f) \in K(\mathscr{H})$ for every $D \otimes f \in \mathcal{A}^{\circ}$. Let $\left(\pi, \rho^{\pi}, \mathscr{H}\right)$ be the unitary representation of $(G, \mathfrak{g})$ that corresponds to $\hat{\pi}$. Theorem 6.4 implies that $\left(\pi, \rho^{\pi}, \mathscr{H}\right)$ is irreducible. The Dixmier-Malliavin theorem implies that there exist $f_{1}, \cdots, f_{r}, h_{1}, \ldots, h_{r} \in \mathcal{D}\left(G_{\varepsilon}\right)$ such that $f=\sum_{i=1}^{r} f_{i} \star h_{i}$. Thus

$$
\hat{\pi}(D \otimes f)=\sum_{i=1}^{r} \hat{\pi}\left(D \otimes f_{i}\right) \hat{\pi}\left(1 \otimes h_{i}\right)=\sum_{i=1}^{r} \hat{\pi}\left(D \otimes f_{i}\right) \pi\left(h_{i}\right) .
$$

From the assumption of the theorem it follows that $\pi\left(h_{i}\right) \in K(\mathscr{H})$ for $1 \leq i \leq r$. Consequently, $\hat{\pi}(D \otimes f) \in K(\mathscr{H})$. 
As in [Salmasian 2010], a Lie supergroup $(G, \mathfrak{g})$ is called nilpotent if $\mathfrak{g}$ is a nilpotent Lie superalgebra.

Theorem 7.2. Let $(G, \mathfrak{g})$ be a connected nilpotent Lie supergroup. Then the $C^{*}$ algebra $\mathcal{A}=\mathcal{A}(G, \mathfrak{g})$ is $C C R$.

Proof. From [Salmasian 2010, Corollary 6.1.1] it follows that the restriction of every irreducible unitary representation $\left(\pi, \rho^{\pi}, \mathscr{H}\right)$ of $(G, \mathfrak{g})$ to $G$ is a direct sum of finitely many irreducible unitary representations. Since every nilpotent Lie group is CCR [Fell 1962], the unitary representation $(\pi, \mathscr{H})$ is completely continuous. Therefore, Theorem 7.1 applies.

Recall from [Neeb and Salmasian 2011] that a Lie supergroup $(G, \mathfrak{g})$ is called $\star$-reduced if for every nonzero $x \in \mathfrak{g}$ there exists a unitary representation $\left(\pi, \rho^{\pi}, \mathscr{H}\right)$ of $(G, \mathfrak{g})$ such that $\rho^{\pi}(x) \neq 0$.

Theorem 7.3. Let $(G, \mathfrak{g})$ be a connected Lie supergroup which is $\star$-reduced and satisfies $\mathfrak{g}_{0}=\left[\mathfrak{g}_{1}, \mathfrak{g}_{1}\right]$. Then the $C^{*}$-algebra $\mathcal{A}=\mathcal{A}(G, \mathfrak{g})$ is $C C R$.

Proof. We show that the hypotheses of Theorem 7.1 are satisfied.

Step 1. From [Neeb and Salmasian 2011, Theorem 7.3.2] it follows that there exists a compactly embedded (in the sense of [Neeb 2000, Definition VII.1.1]) Cartan subalgebra $\mathfrak{t} \subseteq \mathfrak{g}_{\overline{0}}$ and a positive system $\Delta^{+}=\left\{\alpha_{1}, \ldots, \alpha_{r}\right\}$ of $\mathfrak{t}$-roots of $\mathfrak{g}$, such that the space $\mathscr{H}^{\mathrm{t}}$ of $\mathfrak{t}$-finite smooth vectors in $\mathscr{H}$ is a dense subspace of $\mathscr{H}$. Furthermore, $\mathscr{H}^{\mathfrak{t}}$ is an irreducible $\mathfrak{g}$-module which is a direct sum of $\mathfrak{t}$-weight spaces with weights of the form

$$
\lambda-\sum_{i=1}^{r} n_{i} \alpha_{i}, \quad \text { where } n_{i} \in \mathbb{N} \cup\{0\} \text { for every } 1 \leq i \leq r .
$$

Since $\boldsymbol{U}(\mathfrak{g})$ is a finitely generated $\boldsymbol{U}\left(\mathfrak{g}_{\overline{0}}\right)$-module, the irreducible (hence cyclic) $\boldsymbol{U}(\mathfrak{g})$-module $\mathscr{H}^{\mathrm{t}}$ is a finitely generated $\boldsymbol{U}\left(\mathfrak{g}_{\overline{0}}\right)$-module. Since $\boldsymbol{U}\left(\mathfrak{g}_{0}\right)$ is a Noetherian ring [Dixmier 1974, Corollary 2.3.8], $\mathscr{H}^{t}$ is a Noetherian $\boldsymbol{U}\left(\mathfrak{g}_{0}\right)$-module.

Step 2. We prove that $(\pi, \mathscr{H})$ is a direct sum of finitely many irreducible unitary representations of $G$. Assume the contrary. Then we can write $\mathscr{H}=\bigoplus_{\ell=1}^{\infty} \mathscr{H}_{\ell}$ such that each $\mathscr{H}_{\ell}$ is a $G$-invariant closed subspace of $\mathscr{H}$. From the inclusion $\bigoplus_{i=1}^{\infty} \mathscr{H}_{\ell}^{t} \subseteq \mathscr{H}^{t}$ it follows that as a $\boldsymbol{U}\left(\mathfrak{g}_{0}\right)$-module, $\mathscr{H}^{\mathrm{t}}$ is not Noetherian. This contradicts Step 1.

Step 3. From (27) and Step 2 it follows that $(\pi, \mathscr{H})$ is a direct sum of finitely many irreducible highest weight (in the sense of [Neeb 2000, Definition X.2.9]) unitary representations of $G$. From [Neeb 2000, Theorem X.4.10] it follows that every irreducible highest weight unitary representation of $G$ is CCR. Thus $(\pi, \mathscr{H})$ is also a CCR unitary representation of $G$. 
Remark 7.4. Here it would be helpful to the reader to make a correction to [Neeb and Salmasian 2011, Theorem 7.3.2]. The proof of the theorem uses the bijective correspondence between $G$-invariant closed subspaces of $\mathscr{H}$ and $\mathfrak{g}_{0}-$-invariant subspaces of analytic vectors in $\mathscr{H}$. Such a bijection holds only when $G$ is connected. Therefore, connectedness of $G$ should be added in the statement of the theorem. It is plausible to expect that Theorem 7.3 holds when $G$ has finitely many connected components.

Remark 7.5. Let $(G, \mathfrak{g})$ be a connected Lie supergroup such that $\mathfrak{g}$ is a real form of a classical simple Lie superalgebra (see [Musson 2012, Section 1.3]). That is, we assume that $\mathfrak{g} \otimes_{\mathbb{R}} \mathbb{C}$ is isomorphic to one of the Lie superalgebras of type $\mathfrak{s l}(m \mid n)$ for $m>n \geq 0, \mathfrak{p s l}(m \mid m)$ for $m \geq 1, \mathfrak{o s p}(m \mid 2 n)$ for $m, n \geq 0, D(2,1 ; \alpha)$ for $\alpha \neq 0,-1, \mathfrak{p}(n)$ for $n \geq 1, \mathfrak{q}(n)$ for $n \geq 1, G(3)$, or $F(4)$. Assume that $(G, \mathfrak{g})$ has nontrivial unitary representations. (A complete list of these Lie supergroups can be obtained from [Neeb and Salmasian 2011, Theorem 6.2.1].) It is then straightforward to verify that $(G, \mathfrak{g})$ satisfies the hypotheses of Theorem 7.3, and therefore the $C^{*}$-algebra $\mathcal{A}=\mathcal{A}(G, \mathfrak{g})$ is CCR.

\section{Acknowledgements}

During the completion of this project, Salmasian was supported by an NSERC Discovery Grant and the Emerging Field Project "Quantum Geometry" of FAU Erlangen-Nürnberg. The authors thank the referee for useful comments and for pointing out the connection between the recent articles [Alldridge 2014] and [Alldridge et al. 2013] and our work.

\section{References}

[Alldridge 2014] A. Alldridge, "Fréchet globalisations of Harish-Chandra supermodules", preprint, 2014.

[Alldridge et al. 2013] A. Alldridge, J. Hilgert, and M. Laubinger, "Harmonic analysis on HeisenbergClifford Lie supergroups", J. Lond. Math. Soc. (2) 87:2 (2013), 561-585. MR 3046286 Zbl 06160946

[Carmeli et al. 2006] C. Carmeli, G. Cassinelli, A. Toigo, and V. S. Varadarajan, "Unitary representations of super Lie groups and applications to the classification and multiplet structure of super particles", Comm. Math. Phys. 263:1 (2006), 217-258. MR 2006m:22028 Zbl 1124.22007

[Deligne and Morgan 1999] P. Deligne and J. W. Morgan, "Notes on supersymmetry (following Joseph Bernstein)", pp. 41-97 in Quantum fields and strings: A course for mathematicians, I (Princeton, NJ, 1996/1997), edited by P. Deligne et al., Amer. Math. Soc., Providence, RI, 1999. MR 2001g:58007 Zbl 1170.58302

[Dixmier 1974] J. Dixmier, Algèbres enveloppantes, Cahiers Scientifiques 37, Gauthier-Villars, Paris, 1974. MR 58 \#16803a Zbl 0308.17007 
[Dixmier and Malliavin 1978] J. Dixmier and P. Malliavin, "Factorisations de fonctions et de vecteurs indéfiniment différentiables”, Bull. Sci. Math. (2) 102:4 (1978), 307-330. MR 80f:22005 Zbl 0392.43013

[Fell 1962] J. M. G. Fell, "A new proof that nilpotent groups are CCR”, Proc. Amer. Math. Soc. 13 (1962), 93-99. MR 24 \#A3238 Zbl 0105.09602

[Fell and Doran 1988a] J. M. G. Fell and R. S. Doran, Representations of*-algebras, locally compact groups, and Banach * -algebraic bundles, I: Basic representation theory of groups and algebras, Pure and Applied Mathematics 125, Academic Press, Boston, 1988. MR 90c:46001 Zbl 0652.46050

[Fell and Doran 1988b] J. M. G. Fell and R. S. Doran, Representations of ${ }^{*}$-algebras, locally compact groups, and Banach *-algebraic bundles, II: Banach *-algebraic bundles, induced representations, and the generalized Mackey analysis, Pure and Applied Mathematics 126, Academic Press, Boston, 1988. MR 90c:46002 Zbl 0652.46051

[Ferrara et al. 1981] S. Ferrara, C. A. Savoy, and B. Zumino, "General massive multiplets in extended supersymmetry”, Phys. Lett. B 100:5 (1981), 393-398. MR 82f:81085

[Jakobsen 1994] H. P. Jakobsen, The full set of unitarizable highest weight modules of basic classical Lie superalgebras, vol. 111, Mem. Amer. Math. Soc. 532, Amer. Math. Soc., Providence, RI, 1994. MR 95c:17013 Zbl 0811.17002

[Kostant 1977] B. Kostant, "Graded manifolds, graded Lie theory, and prequantization", pp. 177-306 in Differential geometrical methods in mathematical physics (Bonn, 1975), edited by K. Bleuler and A. Reetz, Lecture Notes in Math. 570, Springer, Berlin, 1977. MR 58 \#28326 Zbl 0358.53024

[Musson 2012] I. M. Musson, Lie superalgebras and enveloping algebras, Graduate Studies in Mathematics 131, Amer. Math. Soc., Providence, RI, 2012. MR 2906817 Zbl 1255.17001

[Neeb 2000] K.-H. Neeb, Holomorphy and convexity in Lie theory, de Gruyter Expositions in Mathematics 28, Walter de Gruyter, Berlin, 2000. MR 2001j:32020 Zbl 0936.22001

[Neeb and Salmasian 2011] K.-H. Neeb and H. Salmasian, "Lie supergroups, unitary representations, and invariant cones", pp. 195-239 in Supersymmetry in mathematics and physics, edited by S. Ferrara et al., Lecture Notes in Math. 2027, Springer, Heidelberg, 2011. MR 2012m:22022 Zbl 1275.22011

[Neeb and Salmasian 2013a] K.-H. Neeb and H. Salmasian, "Differentiable vectors and unitary representations of Fréchet-Lie supergroups", Math. Z. 275:1-2 (2013), 419-451. MR 3101815 Zbl 1277.22020

[Neeb and Salmasian 2013b] K.-H. Neeb and H. Salmasian, "Positive definite superfunctions and unitary representations of Lie supergroups", Transform. Groups 18:3 (2013), 803-844. MR 3084335 Zbl 1276.22012

[Neeb et al. 2015] K.-H. Neeb, H. Salmasian, and C. Zellner, "Smoothing operators and C* algebras for infinite dimensional Lie groups", preprint, 2015. arXiv 1505.02659

[Poulsen 1972] N. S. Poulsen, "On $C^{\infty}$-vectors and intertwining bilinear forms for representations of Lie groups”, J. Functional Analysis 9 (1972), 87-120. MR 46 \#9239 Zbl 0237.22013

[Salam and Strathdee 1974] A. Salam and J. Strathdee, "Unitary representations of super-gauge symmetries”, Nuclear Phys. B80 (1974), 499-505. MR 50 \#12029

[Salmasian 2010] H. Salmasian, "Unitary representations of nilpotent super Lie groups", Comm. Math. Phys. 297:1 (2010), 189-227. MR 2011f:22011 Zbl 1192.22003

Received June 4, 2015. Revised July 30, 2015. 
KARL-HERMANN NEEB

DEPARTMENT MATHEMATIK

FAU ERLANGEN-NÜRNBERG

Cauerstr. 11

D-91058 ERLANGEN

GERMANY

neeb@mi.uni-erlangen.de

HADI SALMASIAN

DEPARTMENT OF MATHEMATICS AND STATISTICS

UNIVERSITY OF OTTAWA

585 KING EDWARD AVENUE

OTTAWA, ON K1N 6N5

CANADA

hsalmasi@uottawa.ca 


\title{
PACIFIC JOURNAL OF MATHEMATICS
}

\author{
msp.org/pjm
}

Founded in 1951 by E. F. Beckenbach (1906-1982) and F. Wolf (1904-1989)

\section{EDITORS}

Don Blasius (Managing Editor)

Department of Mathematics

University of California

Los Angeles, CA 90095-1555

blasius@math.ucla.edu

\author{
Paul Balmer \\ Department of Mathematics \\ University of California \\ Los Angeles, CA 90095-1555 \\ balmer@math.ucla.edu \\ Robert Finn \\ Department of Mathematics \\ Stanford University \\ Stanford, CA 94305-2125 \\ finn@math.stanford.edu \\ Sorin Popa \\ Department of Mathematics \\ University of California \\ Los Angeles, CA 90095-1555 \\ popa@math.ucla.edu
}

\author{
Vyjayanthi Chari \\ Department of Mathematics \\ University of California \\ Riverside, CA 92521-0135 \\ chari@math.ucr.edu \\ Kefeng Liu \\ Department of Mathematics \\ University of California \\ Los Angeles, CA 90095-1555 \\ liu@math.ucla.edu \\ Jie Qing \\ Department of Mathematics \\ University of California \\ Santa Cruz, CA 95064 \\ qing@ cats.ucsc.edu
}

\section{PRODUCTION}

Silvio Levy, Scientific Editor, production@msp.org

\section{SUPPORTING INSTITUTIONS}

ACADEMIA SINICA, TAIPEI

CALIFORNIA INST. OF TECHNOLOGY

INST. DE MATEMÁTICA PURA E APLICADA

KEIO UNIVERSITY

MATH. SCIENCES RESEARCH INSTITUTE

NEW MEXICO STATE UNIV.

OREGON STATE UNIV.

\author{
STANFORD UNIVERSITY \\ UNIV. OF BRITISH COLUMBIA \\ UNIV. OF CALIFORNIA, BERKELEY \\ UNIV. OF CALIFORNIA, DAVIS \\ UNIV. OF CALIFORNIA, LOS ANGELES \\ UNIV. OF CALIFORNIA, RIVERSIDE \\ UNIV. OF CALIFORNIA, SAN DIEGO \\ UNIV. OF CALIF., SANTA BARBARA
}

\author{
Daryl Cooper \\ Department of Mathematics \\ University of California \\ Santa Barbara, CA 93106-3080 \\ cooper@math.ucsb.edu \\ Jiang-Hua Lu \\ Department of Mathematics \\ The University of Hong Kong \\ Pokfulam Rd., Hong Kong \\ jhlu@maths.hku.hk \\ Paul Yang \\ Department of Mathematics \\ Princeton University \\ Princeton NJ 08544-1000 \\ yang@math.princeton.edu
}

These supporting institutions contribute to the cost of publication of this Journal, but they are not owners or publishers and have no responsibility for its contents or policies.

See inside back cover or msp.org/pjm for submission instructions.

The subscription price for 2016 is US $\$ 440 /$ year for the electronic version, and $\$ 600 /$ year for print and electronic.

Subscriptions, requests for back issues and changes of subscribers address should be sent to Pacific Journal of Mathematics, P.O. Box 4163, Berkeley, CA 94704-0163, U.S.A. The Pacific Journal of Mathematics is indexed by Mathematical Reviews, Zentralblatt MATH, PASCAL CNRS Index, Referativnyi Zhurnal, Current Mathematical Publications and Web of Knowledge (Science Citation Index).

The Pacific Journal of Mathematics (ISSN 0030-8730) at the University of California, c/o Department of Mathematics, 798 Evans Hall \#3840, Berkeley, CA 94720-3840, is published twelve times a year. Periodical rate postage paid at Berkeley, CA 94704, and additional mailing offices. POSTMASTER: send address changes to Pacific Journal of Mathematics, P.O. Box 4163, Berkeley, CA 94704-0163.

PJM peer review and production are managed by EditFLOW ${ }^{\circledR}$ from Mathematical Sciences Publishers.

\section{PUBLISHED BY}

\section{mathematical sciences publishers \\ nonprofit scientific publishing}

http://msp.org/

(C) 2016 Mathematical Sciences Publishers 


\section{PACIFIC JOURNAL OF MATHEMATICS}

Volume 282 No. $1 \quad$ May 2016

On the half-space theorem for minimal surfaces in Heisenberg space

Tristan Alex

Extending smooth cyclic group actions on the Poincaré homology sphere 9

NIMA ANVARI

A short proof of the existence of supercuspidal representations for all reductive $p$-adic groups

RAPHAËL BEUZART-PLESSIS

Quantum groups and generalized circular elements

MICHAEL BRANNAN and KAY KIRKPATRICK

Volumes of Montesinos links

KathleEn Finlinson and Jessica S. PurCell

Minimal surfaces with two ends which have the least total absolute curvature

SHOICHI FUJIMORI and TOSHIHIRO SHODA

Multiplicité du spectre de Steklov sur les surfaces et nombre chromatique

\section{PIERRE JAMMES}

$E$-polynomial of the $\mathrm{SL}(3, \mathbb{C})$-character variety of free groups

SEAN LAWTON and VicEnTE MuÑOZ

The Blum-Hanson property for $\mathscr{C}(K)$ spaces

PASCAL LEFÈVRE and ÉTIENNE MATHERON

Crossed product algebras and direct integral decomposition for Lie supergroups

KARL-HERMANN NEEB and HADI SALMASIAN Associated primes of local cohomology modules over regular rings

TONY J. PUTHENPURAKAL 